NBSIR 75-695

ULTRASONIC INTERFEROMETER MANOMETER

Peter L.M. Heydemann

National Bureau of Standards

Department of Commerce

Washington, D.C. 20234

\title{
Apri† 1975
}

Interim Report

Prepared for

CCG-Army/Navy

US Army Missile Command

US Army Metrology and Caibration Center

Redstone Arsenal, AL 35809

Naval Plant Representative

Metrology Engineering Center

1675 W. Mission Bivd.

Pomona, CA 91766 


\section{$\underline{\text { ABSTRACT }}$}

This report describes the design of the $15 \mathrm{kPa}$ - U1trasonic Manometer and the associated vacuum equipment. Lay-out drawings are provided. The possibility of using diffusion pump oils as manometric fluid to achieve lower pressures is discussed. Some preliminary thoughts on possible continuation of the project into FY 76 are given.

Key words: Manometer; pressure measurement; ultrasonic interferometer. 



\section{Description of the Manometer and Vacuum Section}

The assembly drawing (Fig. 1) gives a side view of the manometer now under construction on a one to one scale. A large aluminum plate serves as the main support. Three leveling screws are provided. The stainless steel base plate rests on the aluminum plate. It is ground plane and parallel. The titanium disks and the manometer tubes are tied to this steel base. A separate jig plate helps to align the titanium disks. Three stainless steel tubes are welded into the steel plate to connect the columns at the bottom. The connection is made outside the steel plate to allow for the insertion of valves and dampers. The vacuum ports at the top of the columns are connected through welded bellows with the flanges mounted on the shield. The manometer is enclosed with an aluminum shield to improve thermal stability. A thermostated enclosure should surround the entire instrument.

The vacuum system is shown schematically in Fig. 2. Two sets of three welded bellows are used to isoiate the manometer from vibrations carried through the plumbing. Reference vacuum is provided by a trapped, mercury diffusion pump. The line to the fore pump contains a molecular sieve to allow roughing of the system without fear of contaminating the system with oil vapors. The two outer columns are connected to a system containing a trapped fore pump, a volume control for adjustment of pressure and provisions to bleed helium into the system from a compressed gas cylinder. Ports for the connection of instruments to be calibrated are placed near the equalizing valve as close to the manometer as practical. 



\section{Errors caused by Misalignment and Tilt}

Errors in the length measurement with a scale mounted on the manometer column or with the ultrasonic interferometer can occur when the manometer is either misaligned or tilted.

Consider (Fig. 3) the case of a manometer that is misaligned but stable. It is inclined by an angle $\theta$ about an axis through the bottom closures of the outer columns. The true difference in meniscus position is given by

$$
\Delta H=\left[\left(H_{2}^{\prime}-H_{20}^{\prime}\right)-\frac{\left(H_{1}^{\prime}-H_{10}^{\prime}\right)+\left(H_{3}^{\prime}-H_{30}^{\prime}\right)}{2}\right] \cos \theta
$$

where the dashed symbols refer to the measured values.

If the manometer was vertically aligned for the zero reading, but tilted for the pressure measurement and assuming that the center column was offset from the axis AA by $\ell_{2}$ (Fig. 4), we have

$$
\Delta H=\left(H_{2}^{\prime} \cos \theta-H_{0}-l_{2} \sin \theta\right)-\frac{\left(H_{1}^{\prime} \cos \theta-H_{10}\right)+\left(H_{3}^{\prime} \cdot \cos \theta-H_{3 i}\right)}{2}
$$

Finally assume that the manometer was vertically aligned for the zero reading, but tilted about an axis normal to the connecting line $A A$ by an angle $\Theta$. Assume further that the outer columns are spaced $\ell_{1}$ and $\ell_{3}$ from the center column (Fig. 5). We now have

$$
\Delta H=\left(H_{2}^{\prime} \cos \theta-H_{20}\right)-\frac{\left(H_{1}^{\prime} \cos \theta+P_{1} \sin \theta-H_{10}\right)+\left(H_{3}^{\prime} \cos \theta-P_{3} \sin \theta-H_{3 i}\right)}{2}
$$

These are, of course, only examples of the tilt and misalignment errors that one might encounter in practice, and the possible errors have to be carefully considered for each type of manometer.

In the cases considered here the cosine errors are proportional to the true difference in meniscus position. For small angles $\Theta, \cos \theta \sim 1-\frac{\Theta^{2}}{2}$. 



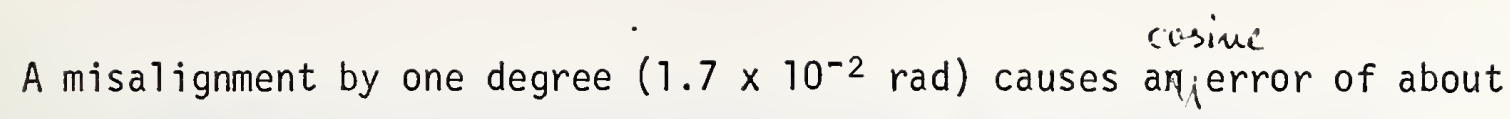
1 in 7000 . A misalignment by five minutes $\left(1.45 \times 10^{-3} \mathrm{rad}\right)$ causes an error of about $1 \mathrm{ppm}$.

However, the terms containing the sine of $\alpha$ in the cases considered here are independent of the difference in meniscus position but are proportional to the offset $\ell$, and are therefore mostly felt at low pressures. In the case considered in Fig. 4 the error is $\ell_{2} \sin$. With $\sin \theta \approx \theta$ and $\ell_{2}=0.5 \mathrm{~mm}$ the error amounts to $8.7 \times 10^{-6} \mathrm{~m}$ for $\mathcal{E}^{E}=1^{\circ}\left(1.7 \times 10^{-2} \mathrm{rad}\right)$ and to $7.3 \times 10^{-7} \mathrm{~m}$ for $\Theta=5^{\prime}\left(1.45 \times 10^{-3} \mathrm{rad}\right)$. A manometer with a resolution of $1.3 \mathrm{mPa}\left(10^{-5}\right.$ torr $)$ and an offset $\ell_{2}$ of $.5 \mathrm{~mm}$ should not tilt more than 4 seconds of arc $\left(2 \times 10^{-5} \mathrm{rad}\right)$.

Such stability is difficult indeed to achieve and only the best possible alignment (small $\ell_{2}$ ) can ensure the expected resolution. We are therefore considering various ways of ensuring near perfect centering of the transducers on the titanium plates and proper alignment of these plates themselves. 



\section{Distortion of the Manometer Base Plate due to Shifting Load}

Since in the ultrasonic manometer al1 column height measurements are made relative to the titanium closure disk, distortion of the disk or of the entire mounting plate is a possible source of error. Measurements were made on an existing mounting plate that is similar to the one to be used in the $10 \mathrm{kPa}-$ Manometer. Fig. 6 gives major dimensions of the plate with its three mercury tubes. The distortion of the mounting plate was measured by placing the feeler rod of an LVDT-transducer at point A. The body of the transformer was mounted on a rigid support placed on the floor. Mercury was alternately moved from the center column to the side columns and back to the center. The average vertical motion of point $A$ due to loading a one meter mercury column into the center tube was $1.3 \times 10^{-7} \mathrm{~m}$. However, the vertical motion of the titanium plates in the outer tubes relative to that in the center tube is expected to be much smaller. Its measurement is being considered for the near future. 


\section{Use of Manometric Fluids other than Mercury}

There are several reasons to use manometric fluids other than mercury. Mercury's high density makes it an ideal fluid for measurements at relatively high pressures, say 10 torr and up. At lower pressures a fluid with lower density might be advantageous, if column length could be determined with the same absolute uncertainty. For example, if visual observation with sighting ring, photo cell etc. or ultrasonic measurements allow for a resolution of the column height measurement of $0.05 \mathrm{~mm}$, the resolved pressure with a mercury column is $6.7 \mathrm{~Pa}\left(5 \times 10^{-2}\right.$ torr $)$ and with a diffusion pump oil column of derisity $0.85 \mathrm{~g} / \mathrm{cm}^{3}$ it is $0.4 \mathrm{~Pa}\left(3 \times 10^{-3}\right.$ torr $)$. A further advantage for some applications is the low vapor pressure of diffusion pump oils $\left(10^{-6} \mathrm{~Pa}, 10^{-8} \mathrm{torr}\right)$ as compared to mercury $\left(10^{-1} \mathrm{~Pa}, 10^{-3}\right.$ torr $)$.

In the ultrasonic manometer the height of the column is deduced from the ultrasonic transit time, speed of sound and ultrasonic absorption are therefore important parameters. The lower the speed of sound is, the higher is the resolution. A low ultrasonic absorption allows use of higher echoes for added resolution.

Some qualitative measurements of speed of sound and attenuation were made. Optimum acoustic match of the ultrasonic transducer to the sample fluids required a redesign of the bottom closure of an interferometer. The new closure is shown in Fig.7. A steel disk with plane and parallel end faces is drilled out to $25 \mathrm{~mm}$ in the center. A single crystal quartz transducer of $19 \mathrm{~mm}$ diameter is placed flush with the top surface of the disk, backed with bakelite and cast in epoxy. A metal layer is evaporated across the surface for electrical contact. The other electrode is brought out through an opening at the lower side. Fig. 8 shows the mounting of this sound projector in the fixed-path interferometer. 

Measurements were first made with water as a test substance. The speed of sound in water measured with this simple device differed only by $0.05 \%$ from the expected value. A variety of diffusion and fore pump oils were then tested. Mineral oils and some silicone oils showed excessive attenuation. Results are shown in Table I. 

TABLE I Properties of Pump Oils

$\begin{array}{lclcc}\text { Type } & \begin{array}{c}\text { Speed of sound } \\ (\mathrm{m} / \mathrm{s})\end{array} & \text { Attenuation } & \begin{array}{c}\text { Density } \\ \left(\mathrm{g} / \mathrm{cm}^{3}\right)\end{array} & \begin{array}{c}\text { Vapor pressure } \\ (\text { torr })\end{array} \\ \text { Speedivac } & - & \text { excessive } & - & - \\ \text { DC } 703 & - & \text { very high } & 1.089 & - \\ \text { DC } 704 & 1430 & \text { high } & 1.066 & 10^{-8} \\ \text { Octoil } & - & \text { very high } & 0.983 & 10^{-7} \\ \text { Octoil S } & 1403 & \text { medium } & 0.912 & 10^{-8} \\ \text { di -butyl pt. } & 1390 & \text { medium } & 1.044 & 10^{-5} \\ \text { Convoil 20 } & - & \text { very high } & 0.86 & 10^{-7} \\ \text { Kinney } & - & \text { very high } & - & -\end{array}$



The attenuation in 0ctoil $S$ is sufficient low to permit operation on the sixth echo with a column length of about $10 \mathrm{~mm}$. In the ultrasonic interferometer this renders a resolution per count at $10 \mathrm{MHz}$ of

$$
\Delta L=\frac{\lambda}{8 p}=\frac{1403}{10^{7} \times 8 \times 6}=2.9 \times 10^{-6} \mathrm{~m}
$$

With the density of $912 \mathrm{~kg} / \mathrm{m}^{3}$ the equivalent pressure is

$$
\Delta_{p}=g \rho \Delta L=9.81 \times 912 \times 2.9 \times 10^{-6}=\left\{\begin{array}{l}
2.6+10^{-2} P_{a} \\
2+10^{-4} \mathrm{tam}
\end{array}\right.
$$

Due to the high viscosity of the oil the reflecting surface of the short oil column will be rather quiet compared to mercury and one might expect to interpolate between counts to 0.1 or 0.05 of a fringe. The ultimate resolution then is $10^{-5}$ torr, well into a range where it might compete with McLeod gages. The ultrasonic manometer thus would be a welcome alternative to the McLeod gage. Both instruments being primary standards it might be attractive to use these instruments together to establish a pressure scale in the medium vacuum range. The electronic instrumentation for ultrasonic manometers using mercury or oil as manometric fluids is essentially the same. 


\section{Possible Future Tasks}

We expect to complete the $10 \mathrm{kPa}$ - ultrasonic manometer by the end of

FY 75. The next task then will be to operate the manometer, evaluate its performance, modify where necessary, and calibrate the instrument. To achieve the target accuracy of $10 \mu \mathrm{mHg}$ at $100 \mathrm{mmHg}$ pressure requires that the temperature of the manometer is stable and known to better than $0.7^{\circ} \mathrm{C}$. It may be advisable at a later date to design a thermal enclosure for the manometer to achieve greater accuracy. This design could be based on our experience with a similar, but much larger enclosure that has recently been constructed.

Barring unforeseen difficulties the $10 \mathrm{kPa}$ - manometer would be completely calibrated, evaluated and documented late in 1975. At that time duplication of the manometer for use in other laboratories should be possible without difficulty.

Since some parts for a second ultrasonic manometer are available we might a consider to develop and construct |manometer for use with a diffusion pump oil to operate at pressures, where it can be compared with a McLeod gage. Cooperation with the Army Metrology Laboratory at Redstone would be most welcome for the purpose of comparison.

Alternatively the second set of parts could be used to assemble a manometer for higher pressures, for example up to $1000 \mathrm{mmHg}$.

The present electronic system for the ultrasonic interferometer is designed to print data on demand. The actual pressure measured by the manometer must then be computed. Since in our present system a microprocessor is used for the control of the electronics, it is feasible to add a computing unit, which, under the control of the microprocessor, will compute the pressure in real time and indicate it in engineering units. 



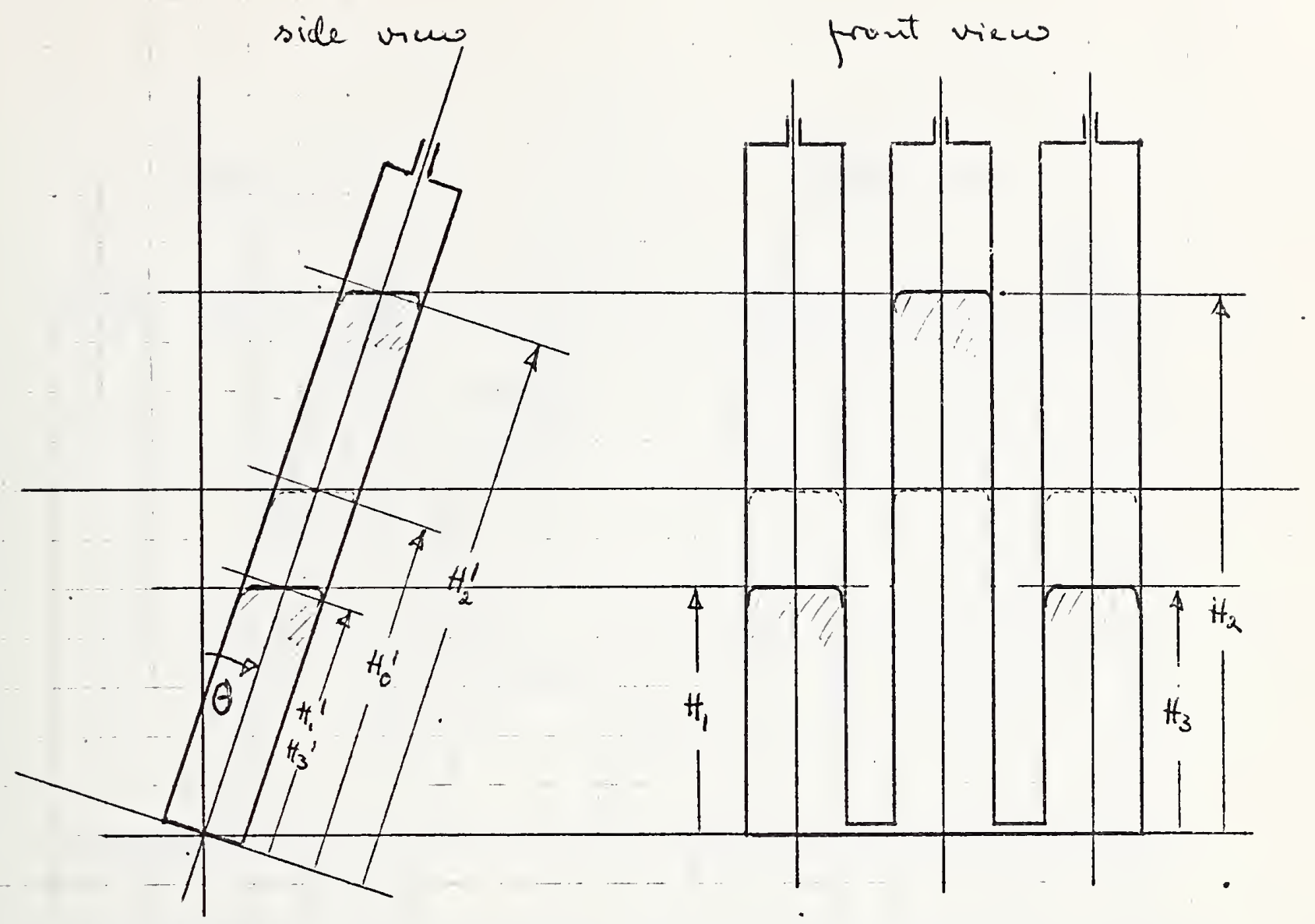

top view

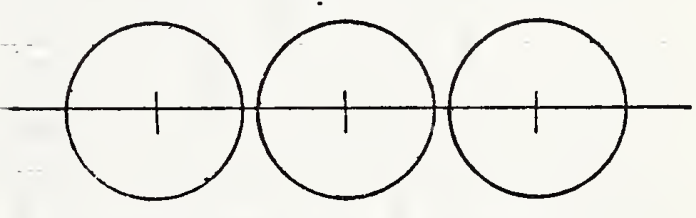

$$
\Delta H=\left[\left(H_{2}^{\prime}-H_{20}^{\prime}\right)-\frac{\left(H_{1}^{\prime}-H_{10}^{\prime}\right)+\left(H_{3}^{\prime}-H_{30}^{\prime}\right)}{2}\right] \cos \theta
$$

Fig. 3 Manometer stable but misaligned about a horizontal axis through the plane of the manonicter tubes 



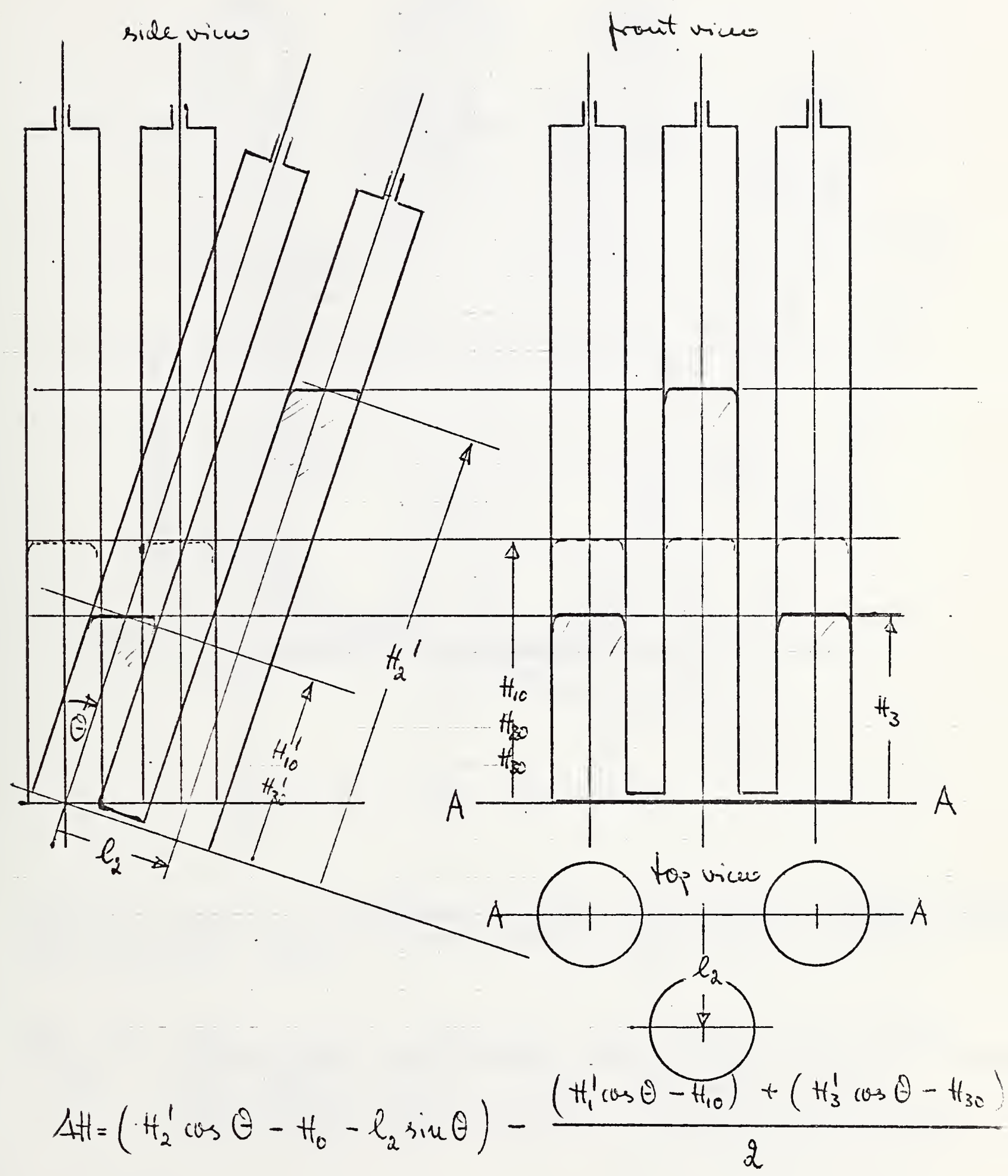

Fig 4 Monomer vertically alignual for zero setting, but tilted as in Fig d 3 for the pressure meriswerent. Center tube attrit from live AA 



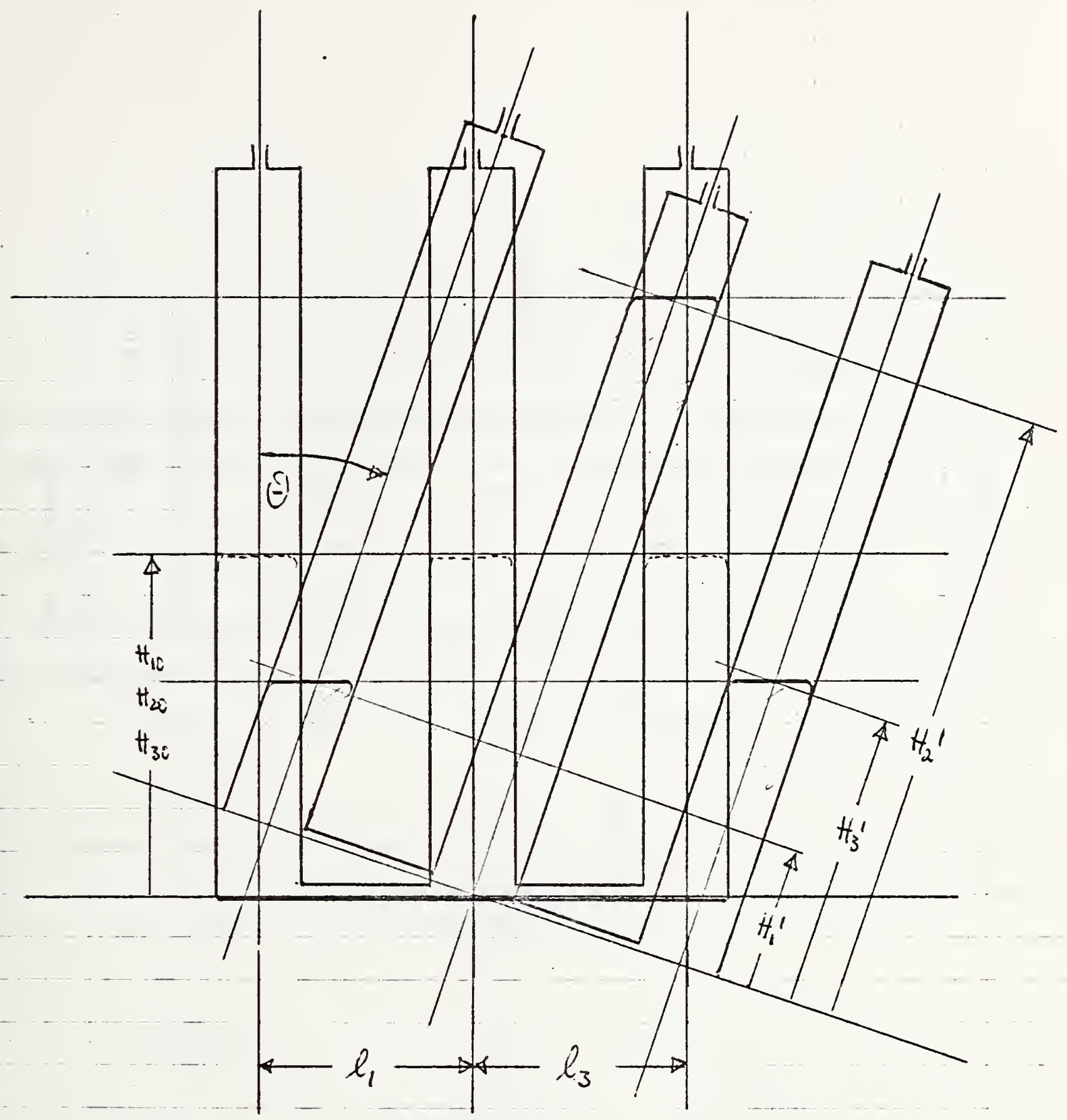

$$
\Delta H=\left(H_{2}^{\prime} \cos \theta-H_{20}\right)-\frac{\left(H_{1}^{\prime} \cos \theta+l_{1} \sin \theta-H_{10}\right)+\left(H_{3}^{\prime} \cos \theta-l_{3} \sin \theta-H_{30}\right)}{2}
$$

Fig. 5 Manometer vertically aligned for zero retting, but tilled about an axis nornach to the nuchometer plane for the pressure measurement. 



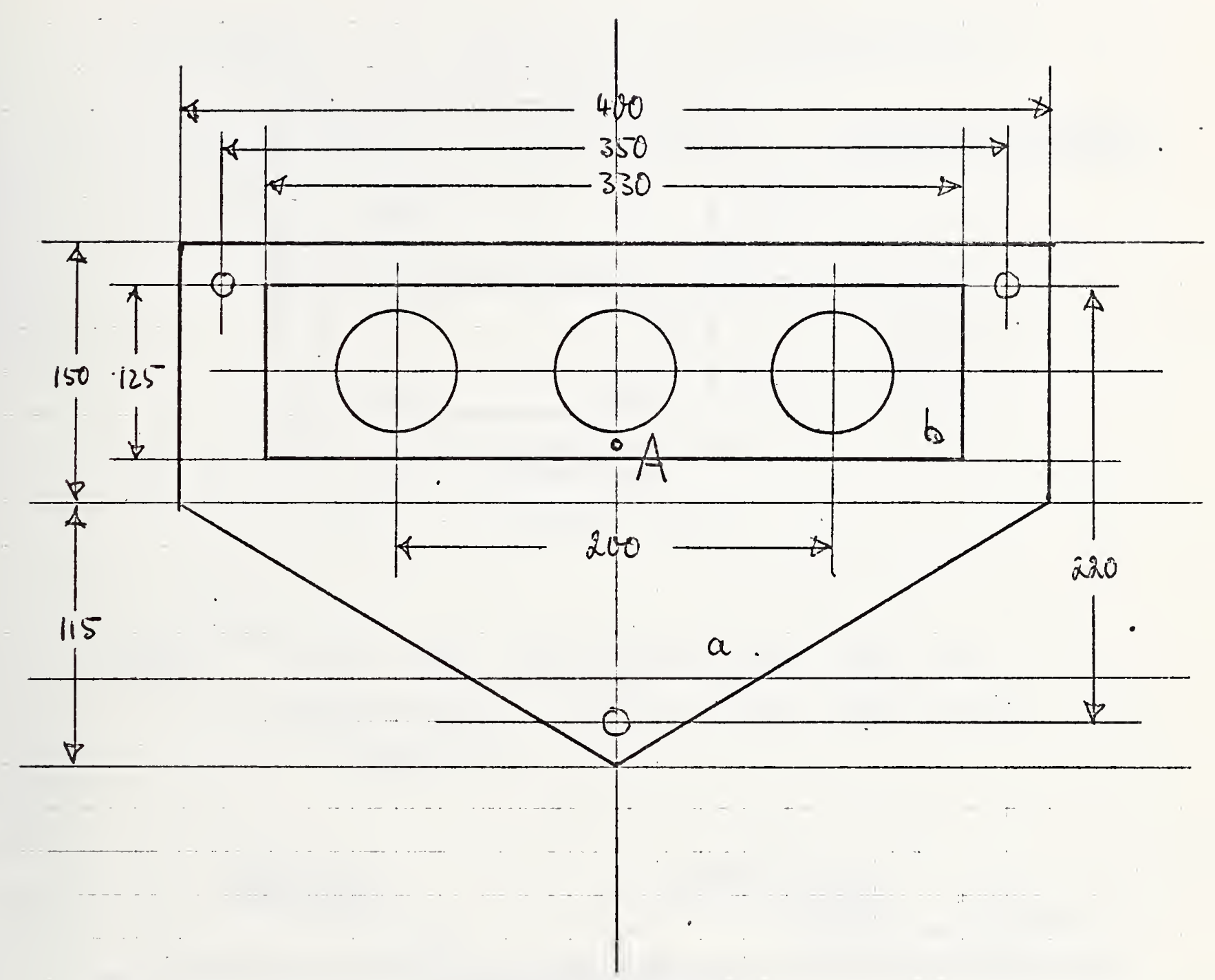

Fig 6 Manometer Base Plank (a) and Mounting Plate (b). Each plate is uncle from stainleas stab and is 25 mun this?

LVDT-feeler rod was place l at A. Dimensions in [min] 



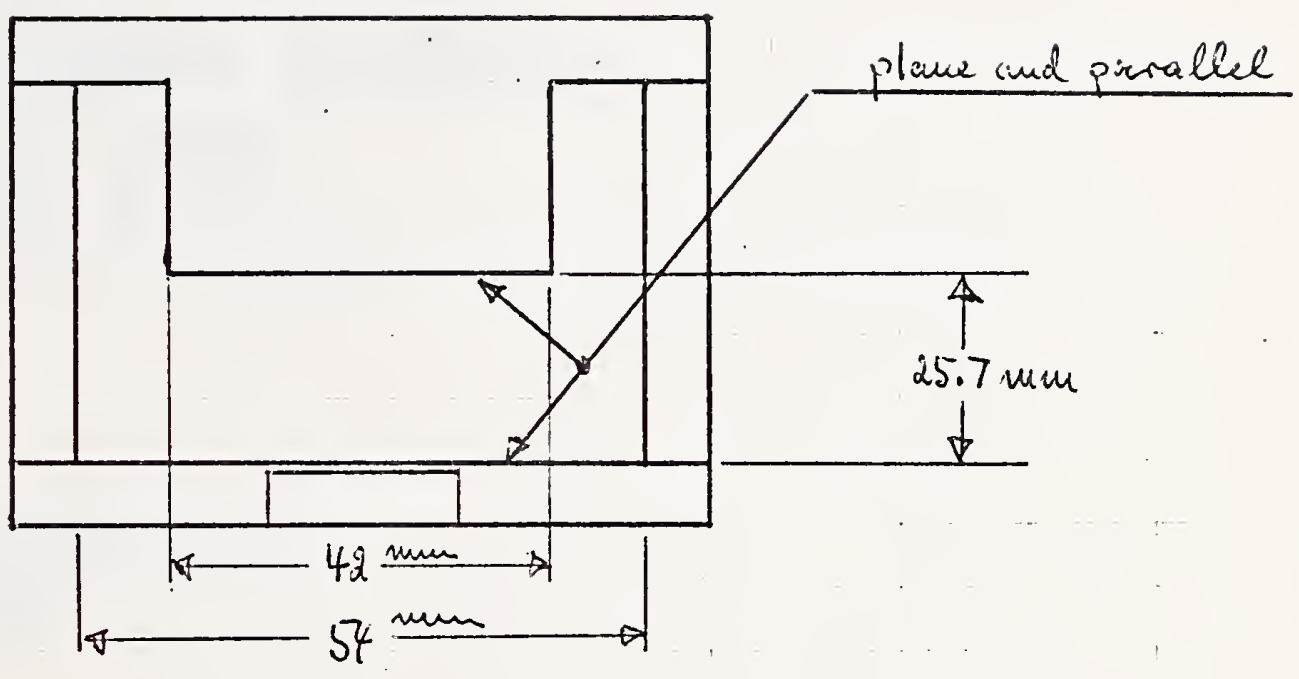

Figs Fixcil path interferomeler for the mecasurement of the speed of round in liquids.

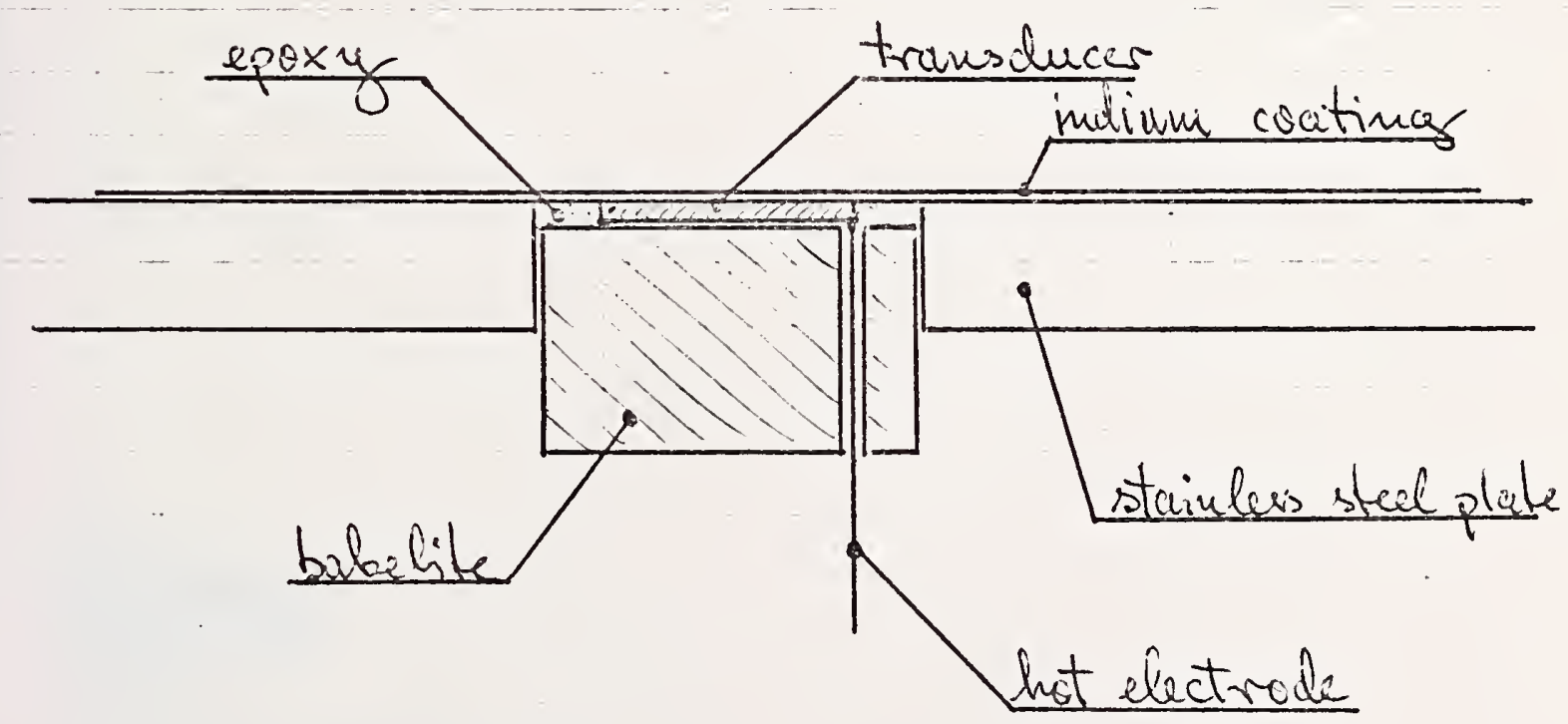

Fig.7 Encapsulated, frout-plane ultrarsonic transducer assembly 
